\title{
Materials Science Applications and Analysis of Very Large 4D-STEM Experiments
}

Colin Ophus ${ }^{1}$, Benjamin Savitzky ${ }^{1}$, Philipp Pelz ${ }^{2}$, Alexander M Rakowski ${ }^{3}$, Luis Rangel DaCosta ${ }^{4}$, Lauren Hughes ${ }^{5}$, Steven Zeltmann ${ }^{4}$, Karen C Bustillo ${ }^{5}$, Mary Scott ${ }^{2}$ and Andrew Minor ${ }^{2}$

${ }^{1}$ Lawrence Berkeley National Laboratory, California, United States, ${ }^{2}$ UC Berkeley, Berkeley, California, United States, ${ }^{3}$ LBNL, United States, ${ }^{4}$ UC Berkeley, United States, ${ }^{5}$ Lawrence Berkeley National Laboratory, United States

With the introduction of high speed direct electron detectors, scanning transmission electron microscopy (STEM) can now record full images of the diffracted electron probe scanned over the sample, producing a four-dimensional dataset, we refer to as a 4D-STEM experiment [1]. Figure 1a shows the experimental geometry of 4D-STEM measurements, using conventional apertures, bullseye patterned apertures to enhance strain measurements [2], and multibeam apertures which enable multiple simultaneous diffraction experiments [3]. These diffraction images of the electron probe are extremely rich in atomic-scale information, such as the sample structure, phase, orientation, composition, presence of defects, and more. The STEM probe size can vary over multiple orders of magnitude, ranging from sub-atomic to tens of nanometers, allowing measurements ranging from the position of single atoms to robust statistical measurements of properties such as lattice spacing over many crystalline unit cells. Because the size of a STEM probe is decoupled from the step size between measurements, we can tune the field of view of a given measurement to any desired length scales. The high speed of modern detectors has made recording up to a million diffraction images per dataset routine, requiring new approaches and software tools to deal with this "data deluge."

Here, I will discuss several 4D-STEM applications in materials science. These include the example shown in Figure $1 \mathrm{~b}$, where diffraction patterns were collected over a large interfacial region of a solid state battery sample, generating a statistically robust picture of the sample. I will discuss atomic-resolution imaging of a complex nanotube-nanowire hybrid sample, shown in Figure 1c, which was reconstructed using a scattering matrix (S-matrix) phase contrast inversion method [4]. I will also show results from a new characterization method called Bragg interferometry, applied to 2D material heterostructures. Figure 1d shows the application of this method to strain field measurements in twisted bilayer graphene on a BN substrate [5]. For each of these applications, I will discuss how we optimized the measurement parameters and performed the data analysis, often using our open source 4D-STEM analysis toolkit called py4DSTEM [6]. I will also demonstrate some new 4D-STEM simulation methods that are orders of magnitude faster, and with negligible differences in accuracy. Finally, I will also discuss our current efforts to deploy Machine Learning methods in order to perform automated, robust and accurate 4D-STEM measurements [7]. 

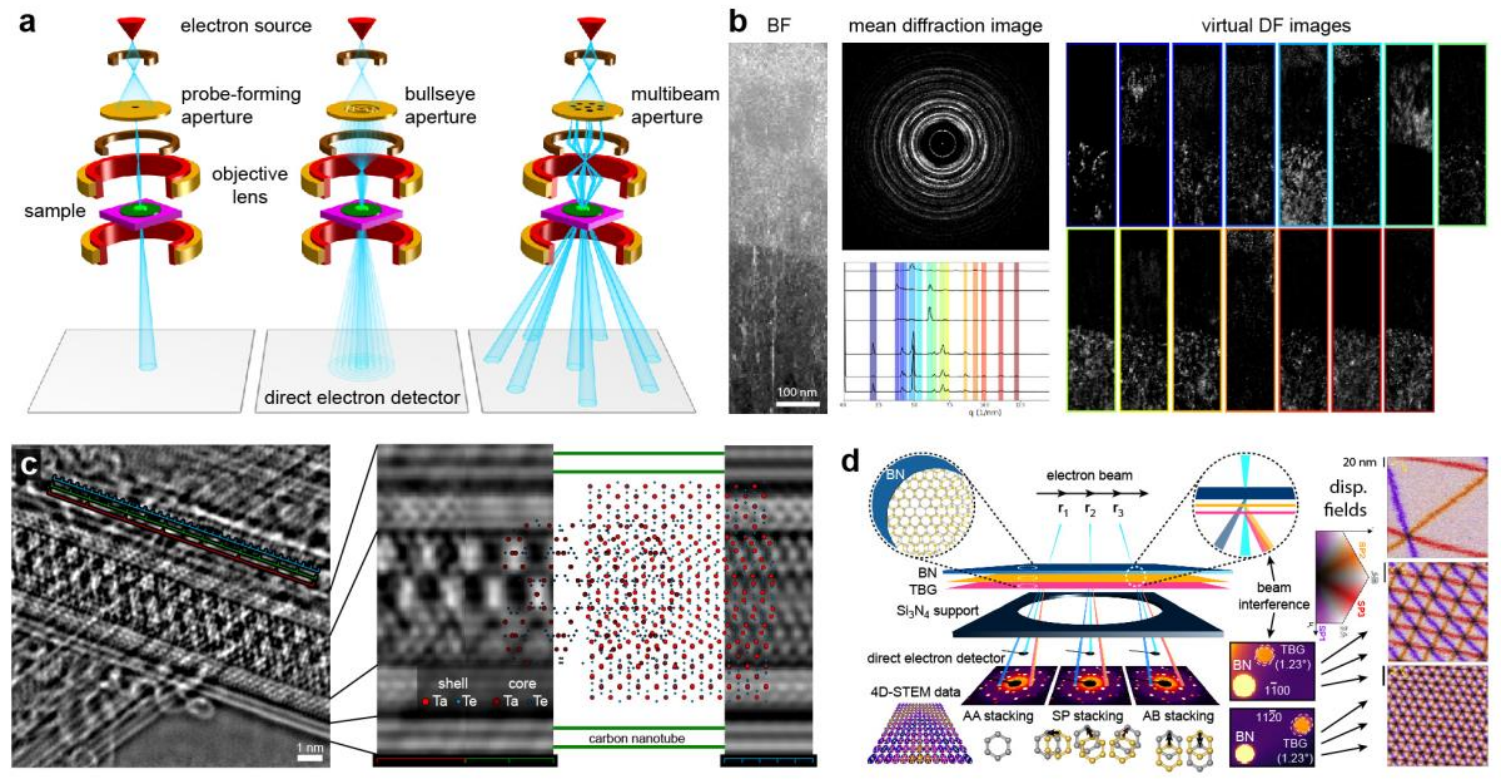

Figure 1. Applications of 4D-STEM in Materials Science. (a) From left to right, the experimental geometry of a conventional nanobeam diffraction experiment, a nanobeam experiment using a patterned bullseye aperture, and a multibeam diffraction experiment. (b) 4D-STEM experiment on a solid state battery interface, showing a virtual bright field (BF) image, the mean diffraction signal, mean radial diffraction signal, and virtual dark field (DF) images formed from different annular apertures. (c) Scattering matrix reconstruction phase contrast imaging of a carbon nanotube containing a complex nanowire structure, adapted from [4]. 4D-STEM Bragg interferometry used to measure strain fields of bilayer graphene on a BN substrate, adapted from [5].

\section{References}

[1] C Ophus, Microscopy and Microanalysis 25, 563 (2019).

[2] SE Zeltmann et al., Ultramicroscopy 209. 112890 (2020).

[3] X Hong et al., Microscopy and Microanalysis 1, (2020).

[4] P Pelz et al., arXiv:2008.12768 (2020).

[5] NP Kazmierczak et al., arXiv:2008.09761 (2020).

[6] B Savitzky et al., arXiv:2003.09523 (2020).

[7] Work at the Molecular Foundry was supported by the Office of Science, Office of Basic Energy Sciences, of the U.S. Department of Energy under Contract No. DE-AC02-05CH11231. 